\title{
ARTICLE \\ Kinetics of oxytocin effects on amygdala and striatal reactivity vary between women and men
}

\author{
Jana Lieberz ${ }^{1}$, Dirk Scheele (iD) ${ }^{1}$, Franny B. Spengler ${ }^{2}$, Tatjana Matheisen ${ }^{1}$, Lìa Schneider ${ }^{1}$, Birgit Stoffel-Wagner ${ }^{3}$, Thomas M. Kinfe ${ }^{4}$ and \\ René Hurlemann ${ }^{1,5,6}$
}

\begin{abstract}
Accumulating evidence suggests that intranasal oxytocin (OXT; 24 IU) reduces amygdala responses to fear-related stimuli in men, while exerting inverse effects in women. However, OXT enhances activity of the brain reward system in both sexes. Importantly, a crucial and still open question is whether there are sex-specific dose-response relationships for the amygdala and striatal regions. To address this question, a total of 90 healthy women participated in a double-blind, placebo-controlled crossover functional magnetic resonance imaging (fMRI) study and the results were compared with our previous findings from men. Participants were randomly assigned to three doses of OXT (6 IU, $12 \mathrm{IU}$, and $24 \mathrm{IU})$ and completed an emotional face recognition task including fearful and happy faces of varying emotional intensities. Across doses, OXT enhanced amygdala reactivity to low fearful faces compared to placebo and increased responses to happy faces in the dorsal striatum in women. While treatment effects on amygdala reactivity were evident at each given dose, the OXT effect on striatal responses to social stimuli was more pronounced with higher doses, but this dose-dependent effect did not survive correction for multiple comparisons. Importantly, OXT effects on amygdala and striatal activation significantly differed between sexes and striatal baseline sexual-dimorphic response patterns were diminished after administration of OXT. Our findings suggest that OXT increases the salience of social signals by strengthening the sensitivity for these signals in the amygdala and in the striatum in women, while OXT may primarily induce anxiolysis by reducing amygdala responses in men.
\end{abstract}

Neuropsychopharmacology (2020) 45:1134-1140; https://doi.org/10.1038/s41386-019-0582-6

\section{INTRODUCTION}

The hypothalamic peptide oxytocin (OXT) is a key modulator of social cognition and behaviors including fear learning [1, 2], stress responses [3-5], pair-bonding [6], and parent-infant attachment [7]. In both human and animal studies, OXT has been found to reduce threat-induced reactivity of the amygdala [1, 8-13]. Furthermore, current perspectives on the prosocial effects of OXT emphasize that the peptide enhances the salience of social signals by modulating the activation of the brain reward system [14-17]. This dual mechanism of action renders OXT a promising system for the treatment of various mental disorders associated with impaired social interaction abilities, such as autism spectrum or anxiety disorders $[18,19]$. However, the rapid translation into clinical efficacy is currently hampered by limited insights into the dose-response relationships of OXT. We have recently shown that a dose of 24 international units (IU) appears optimal for inhibiting amygdala responses to social fear signals in healthy men [20]. However, there is accumulating evidence that the same dose causes the opposite effect in women [9, 21, 22]. Similarly, sexualdimorphic anxiolytic effects have been observed in mice [23] and rats [24]. Possible sex-specific OXT effects on the brain reward system are less consistent, but have also been observed. While
OXT enhances activation in reward-associated brain regions in response to the romantic partner in both sexes [14-16], it selectively increases striatal reactivity to reciprocated cooperation in men but not in women [25-29].

Further differential effects of OXT in women and men have been documented in various domains. For instance, in women, 24 IU of OXT improved the ability to recognize familial relationships [30], biased them to respond more altruistically [31], and increased the magnitude of perceived support during recall of negative autobiographical memories in a social context [32]. In men, 24 IU of OXT facilitated the accurate perception of competition, promoted self-interest moral judgements and had no significant effect on recalling emotional autobiographical memories. Given this empirical background, one might assume that OXT may have evolved to prompt affiliative efforts aimed at restoring positive social contacts in women ("tend-and-befriend" model) [33], but may primarily affect fight-or-flight responses via anxiolysis in men.

However, little is known about the basic mechanisms underlying these sexual-dimorphic effects. OXT might interact with gonadal hormones such as progesterone and estradiol, whose levels differ between women and men and which are implicated in the neural processing of emotional stimuli [34, 35]. Furthermore,

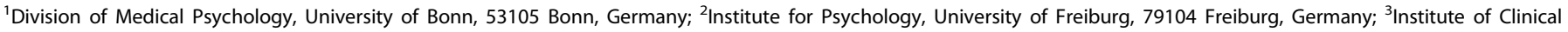

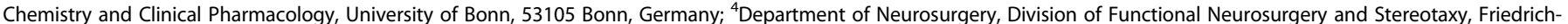

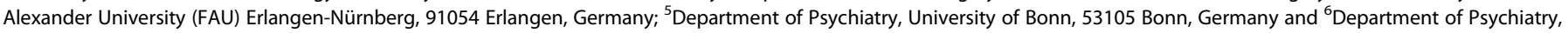
University of Oldenburg Medical Campus, 26160 Bad Zwischenahn, Germany

Correspondence: Dirk Scheele (Dirk-Scheele@gmx.de)

These authors contributed equally: Jana Lieberz, Dirk Scheele.

Received: 21 August 2019 Revised: 20 November 2019 Accepted: 25 November 2019

Published online: 30 November 2019 
OXT binding sites are widely distributed in the brain, and animal studies suggest that OXT receptor densities differ between sexes in various brain regions interconnected with the amygdala or striatal regions (e.g. the bed nucleus of the stria terminalis) $[36,37]$.

Importantly, however, higher doses of intranasal OXT (48 IU) tend to enhance amygdala reactivity to fearful stimuli in men [20], indicating an inverted- $U$ relationship between the amount of administered OXT and anxiolytic effects on threat-induced amygdala activation. Assuming this inverted-U dose-response relationship is also true for women, sex-specific effects of OXT treatments might also be a byproduct of a shifted dosedependent target engagement in women, initiated at lower doses compared to men $[28,38]$. We thus hypothesized that lower doses might be needed in women to reveal a decrease in amygdala reactivity as observed in men after the administration of 24 IU of OXT.

Therefore, the present randomized, double-blind, placebocontrolled crossover study aims to investigate potential dosedependent effects of intranasal OXT on amygdala and striatal responses to emotional faces in healthy women and to validate the sex-specificity of OXT effects. A total of 92 healthy, righthanded women were randomly assigned to three doses of OXT (24 IU, $12 \mathrm{IU}$, and $6 \mathrm{IU}$ ). The rationale of focusing on lower rather than higher doses was based on evidence for higher baseline endogenous OXT levels in women compared to men [39], such that lower doses might be needed to produce anxiolytic effects in women. We thus hypothesized that 24 IU of intranasal OXT would increase amygdala responses to fearful stimuli in women, whereas lower doses (6 IU or $12 \mathrm{IU}$ ) would inhibit these responses. Since an inverted-U relationship has also been hypothesized between OXT dose and reward-associated brain activation [28], and given evidence for enhanced brain reward system activity after administration of 24 IU intranasal OXT in women [15, 16], we predicted heightened striatal responses to positive social stimuli after OXT administration and further hypothesized that this effect would be more pronounced after 24 IU compared to lower doses (6 IU or $12 \mathrm{IU}$ ). Finally, we hypothesized that OXT effects after administration of equivalent doses would significantly differ between sexes, whereas effects of lower doses of OXT in women $(6,12$, and $24 \mathrm{IU})$ would mirror the effects of higher doses of OXT in men $(12,24$, and $48 \mathrm{IU}$, respectively).

\section{MATERIALS AND METHODS}

Experimental design and participants

We used a randomized, double-blind, placebo-controlled crossover design. Out of a total of 105 participants who were invited to a screening session prior to the testing sessions, 92 healthy, righthanded women were eligible for enrollment and randomly assigned to three groups differing in doses of administered treatment: $6 \mathrm{IU}(n=31), 12 \mathrm{IU}(n=31)$, and $24 \mathrm{IU}(n=30$; for details, see Supplementary Materials and Methods). The study was approved by the local ethics committee of the Medical Faculty of the University of Bonn, Germany, and carried out in accordance with the latest revision of the Declaration of Helsinki. All participants provided written informed consent and were free of current physical or psychiatric illness as assessed by self-report and the Mini-International Neuropsychiatric Interview (MINI [40]) in the screening session. None of the participants used hormonal contraceptives or was pregnant while taking part in the study. Following the screening session, participants underwent two identical testing sessions on separate days (one session after placebo [PLC] and one session after OXT administration), scheduled at least one month apart. The order of substance administration was randomized across participants. Two participants were excluded after the first testing session as they no longer fulfilled inclusion criteria (see Supplementary Materials and
Methods), resulting in 90 participants (mean age $\pm S D=23.9 \pm 4.4$ years) for the analyses ( $n=30$ in each dose group). Furthermore, 11 participants were excluded from functional magnetic resonance imaging (fMRI) analyses due to technical malfunctions $(n=$ 2) or excessive head motion ( $>4 \mathrm{~mm} /{ }^{\circ} ; n=9$ ). Thus, the final sample for $\mathrm{fMRI}$ analyses consisted of 79 participants (mean age \pm $\mathrm{SD}=24.0 \pm 4.3$ years; $6 \mathrm{IU}: n=28,12 \mathrm{IU}: n=26,24 \mathrm{IU}: n=25$ ). There were no a priori differences between dose groups regarding demographic and psychological measurements (see Table S1).

\section{Testing sessions}

Participants completed two testing sessions, both including an fMRI scan and questionnaires measuring mood and state anxiety at the beginning and end of the testing session (the Positive and Negative Affect Schedule, PANAS [41] and the State-Trait Anxiety Inventory, STAI [42]; see Table S2). After completion of the questionnaires, participants self-administered 6,12 , or $24 \mathrm{IU}$ of synthetic OXT (depending on dose group; Sigma-Tau Industrie Farmaceutiche Riunite S.p.A., Rome, Italy) or PLC via nasal spray under the supervision of a trained research assistant and in accordance with the latest standardization guidelines [43] (for details, see Supplementary Materials and Methods). The PLC solution contained identical ingredients except for the peptide itself. Imaging data were collected $45 \mathrm{~min}$ after substance administration while participants completed an emotional face recognition task [20]. Briefly, participants were instructed to decide via button press whether morphed face stimuli displayed a neutral, fearful, or happy expression of varying emotional intensities (neutral, low and high fearful, low and high happy) (for details, see [20]). Blood was sampled before treatment administration (baseline) and immediately after fMRI scanning. All participants were tested in the luteal phase of their menstrual cycle as assessed by self-report and validated by blood assays (estradiol and progesterone concentrations; see Supplementary Materials and Methods).

\section{fMRI data acquisition and analysis}

Imaging data were recorded on a $1.5 \mathrm{~T}$ Siemens Avanto MRI system (Siemens, Erlangen, Germany) using an amygdala-sensitive sequence with an echo time (TE) decreased linearly by $10 \mathrm{~ms}$ between slice 9 and slice 14, resulting in a final TE of $40 \mathrm{~ms}$ [44]. Data were preprocessed (see Supplementary Materials and Methods) and analyzed using standard procedures in SPM8 software (Wellcome Trust Centre for Neuroimaging, London, UK) implemented in MATLAB 7.11 (The Mathworks, Inc., Natick, MA, USA) as in our previous study [20]. A two-stage approach based on the general linear model implemented in SPM8 was used for statistical analyses. For region of interest analyses, the amygdala and striatal regions (putamen, caudate nucleus, globus pallidus, and nucleus accumbens) were anatomically defined according to the Wake Forest University Pick Atlas $[45,46]$. $P$ values $<0.05$ after correction for multiple comparisons (familywise error, FWE) were considered significant. Finally, to examine possible dose-dependent effects, parameter estimates of significant OXT effects on amygdala and striatal responses to fearful or happy faces relative to neutral faces were averaged across all voxels of anatomically defined regions of interest, extracted, and analyzed in SPSS 24 (IBM Corp., Armonk, NY, USA) using mixed analyses of variance (ANOVAs) and Bonferroni-corrected ( $p_{\text {corr }}$ ) post hoc $t$-tests. We also explored whole brain OXT effects across all participants and dose groups (for details, see Supplementary Materials and Methods).

Behavioral, demographic, and neuroendocrine data were analyzed using standard procedures in SPSS 24 including mixed ANOVAs and $t$-tests. Greenhouse-Geisser correction was applied if the assumption of sphericity was significantly violated as assessed with Mauchly's test. Partial eta-squared and Cohen's $d$ were calculated as measures of effect size. To examine whether 
individual characteristics of the participants moderate the effectiveness of OXT, we conducted explorative Pearson's product-moment correlations between OXT effects and behavioral, demographic, psychological, and neuroendocrine measurements. For results of behavioral data and correlation analyses, see Supplementary Results.

To test the hypothesis of sexual-dimorphic OXT effects, parameter estimates of significant OXT effects in the female sample and the sample of our previous study with healthy men [20] were analyzed using mixed ANOVAs with the additional between-subject factor sex and two-sample $t$-tests. For details of comparative analyses, see Supplementary Materials and Methods.

Data analysis was preregistered prior to conducting any analyses (https://osf.io/yxrsf/).

\section{RESULTS}

Neuroendocrine parameters

Estradiol, progesterone, progesterone/estradiol ratio, and OXT levels at baseline differed neither between dose groups nor between OXT and PLC testing sessions (see Table S3). No interaction between testing session and dose group was found (all $F s<1.90$, all $p s>0.15$ ).

A mixed ANOVA with the within-subject factor treatment (OXT, PLC), the between-subject factor dose group (6 IU, $12 \mathrm{IU}, 24 \mathrm{IU})$, and the pre-post difference in OXT plasma concentrations revealed main effects of treatment $\left(F_{(1,85)}=338.00, p<0.001\right.$, $\left.\eta_{p}{ }^{2}=0.80\right)$ and dose group $\left(F_{(2,85)}=23.76, p<0.001, \eta_{p}{ }^{2}=0.36\right)$. Furthermore, a significant interaction between treatment and dose group indicated a difference in the magnitude of OXT increase between dose groups $\left(F_{(2,85)}=16.16, p<0.001, \eta_{p}{ }^{2}=\right.$ $0.28)$. Post hoc tests revealed that the plasma OXT concentration was significantly increased after OXT administration in all dose groups (all $t \mathrm{~s}>11.51$, all $p \mathrm{~s}_{\text {corr }}<0.001$, all $d \mathrm{~s}>3.25$ ), but increases in OXT levels after $24 \mathrm{IU}$ and $12 \mathrm{IU}$ of OXT in contrast to PLC were significantly greater compared to $6 \mathrm{IU}$ (24 IU vs. $6 \mathrm{IU}: t_{(56)}=5.63$, $p_{\text {corr }}<0.001, d=1.48 ; 12$ IU vs. 6 IU: $t_{(43.27)}=5.16, p_{\text {corr }}<0.001$, $d=1.34)$, whereas there was no difference between the 12 and 24 IU groups $\left(t_{(57)}=0.06, p_{\text {corr }} \approx 1.00\right.$; see Fig. 1$)$.

fMRI results

OXT effects on amygdala responses. Across all participants and dose groups, OXT significantly enhanced right amygdala responses to low fearful (i.e. [OXT low fearful $>$ neutral $]>[P L C$ low fearful > neutral]; Montreal Neurological Institute [MNI] peak coordinates $x, y, z: 30,-8,-12, t_{(78)}=3.64, k=135, p_{\mathrm{FWE}}=0.02$, see Fig. 2a) but not to high fearful faces (MNI peak coordinates $x, y, z$ :

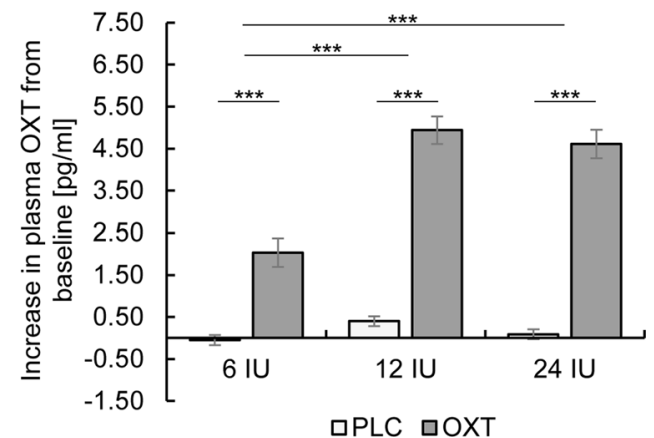

Fig. 1 Changes in plasma oxytocin (OXT) concentration after OXT and placebo (PLC) administration relative to baseline. Administration of 12 and 24 international units (IU) of OXT induced a significantly greater increase in OXT levels than $6 \mathrm{IU}$. Error bars indicate standard errors of the mean. Abbreviations: corr, Bonferroni-corrected; IU, international units; OXT, oxytocin; PLC, placebo. ${ }^{* * *} p_{\text {corr }}<0.001$. $\left.28,-2,-16, t_{(78)}=2.39, k=25, p_{\mathrm{FWE}}=0.25\right)$. No significant effects of OXT on left amygdala responses to low or high fearful faces or to fearful faces across emotional intensities were found (all $t s<2.40$, all FWE-corrected $p s>0.22$ ). Furthermore, we observed no dampening effects on amygdala activation (e.g. [OXT fearful $>$ neutral $]<$ $[P L C$ fearful > neutral]) or significant OXT effects on amygdala responses to happy faces. Mixed ANOVAs with treatment (OXT, PLC) and emotion (e.g. neutral, fearful) as within-subject factors, dose group (6 IU, $12 \mathrm{IU}, 24 \mathrm{IU})$ as between-subject factor, and extracted parameter estimates averaged across right amygdala voxels as dependent variables were calculated to further investigate OXT effects on amygdala activation. Importantly, significant interactions of treatment and emotion confirmed OXT effects on responses to fearful $\left(F_{(1,76)}=7.03, p=0.01, \eta_{p}{ }^{2}=0.09\right)$ and low fearful faces $\left(F_{(1,76)}=6.70, p=0.01, \eta_{p}^{2}=0.08\right.$; see Fig. $\left.2 a\right)$ in contrast to neutral faces, whereas no interaction of treatment and emotion was observed for amygdala responses to high fearful faces $\left(F_{(1,76)}=1.86\right.$, $p=0.18$ ). Post hoc tests revealed significantly greater amygdala responses to fearful $\left(t_{(78)}=4.14, p_{\text {corr }}<0.001, d=0.15\right)$ and low fearful faces $\left(t_{(78)}=3.75, \quad p_{\text {corr }}<0.001, d=0.15\right.$; see Fig. 2a) compared to neutral faces after OXT administration while no other post-hoc comparison reached significance (all $t \mathrm{~s}\left\langle 1.86\right.$, all $\left.p \mathrm{~s}_{\text {corr }}\right\rangle$ 0.27). For further analyses of OXT effects on amygdala responses, see Supplementary Results.

OXT effects on striatal reactivity. OXT significantly enhanced activation in response to happy faces (i.e. [OXT happy $>$ neutral] $>$ $[\mathrm{PLC}$ happy > neutral]) across all dose groups in the right putamen (MNI peak coordinates $x, y, z: 34,-18,-4, t_{(78)}=3.83, k=45, p_{\mathrm{FWE}}=$ $0.03)$. Mixed ANOVAs with extracted parameter estimates averaged across all right putamen voxels as dependent variables confirmed a significant treatment multilpy emotion interaction for putamen responses across emotional intensities $\left(F_{(1,76)}=6.42, p=0.01, \eta_{p}^{2}\right.$ $=0.08)$ and for low happy faces $\left(F_{(1,76)}=9.13, p=0.003, \eta_{\mathrm{p}}{ }^{2}=0.11\right.$; see Fig. 2c). Post-hoc tests indicated enhanced putamen reactivity to low happy compared to neutral faces after OXT administration $\left(t_{(78)}=2.38, p=.02\left[p_{\text {corr }}=0.08\right.\right.$ ]; see Fig. 2 c; all other $t s<1.67$, all $\left.p \mathrm{~s}_{\text {corr }}>0.40\right)$. In contrast, no significant interaction of treatment and emotion was found for putamen responses to high happy faces $\left(F_{(1,76)}=2.43, p=0.12\right)$. Therefore, we decided to focus on right putamen reactivity to happy faces across emotional intensities and to low happy faces. Additional analyses of putamen responses to happy faces and further results of OXT effects on striatal reactivity are reported in the Supplementary Results.

Dose-dependent effects. Importantly, no dose-dependent effects (main effect of dose or interactions with dose group) were found for right amygdala responses to fearful faces across emotional intensities (all $F s<1.24$, all $p s>0.29$ ) or low fearful faces (all $F s<$ 0.72 , all $p s>0.49$; see Fig. $2 b$ ), indicating that OXT effects did not significantly differ between dose groups.

In contrast, trend-to-significant interactions of treatment and dose group were found for right putamen reactivity, implying dose-dependent OXT effects on responses to social stimuli across happy and neutral $\left(F_{(2,76)}=3.08, p=0.05, \eta_{p}{ }^{2}=0.08\right)$ as well as low happy and neutral faces $\left(F_{(2,76)}=3.12, p=.05, \eta_{p}{ }^{2}=0.08\right.$; see Fig. 2d). Post hoc tests disentangling the potential dosedependent OXT effects on right putamen activation are reported in the Supplementary Results. No other dose-dependent effects (main effect of dose or interactions with dose group) were found for right putamen reactivity to happy or low happy faces (all $\mathrm{Fs}<$ 2.35, all $p s>0.10)$.

Whole brain. OXT significantly enhanced responses to happy stimuli ([OXT happy $>$ neutral $]>[\mathrm{PLC}$ happy $>$ neutral $]$ ) in the left precuneus (MNI peak coordinates $x, y, z:-4,-68,30, t(78)=4.34, k=$ $144, p_{\mathrm{FWE}}=0.02$; see Fig. 3 ). We found no evidence for effects on 
A

(Across doses: OXT Low Fearful $>$ Neutral $>$ PLC Low Fearful $>$ Neutral )
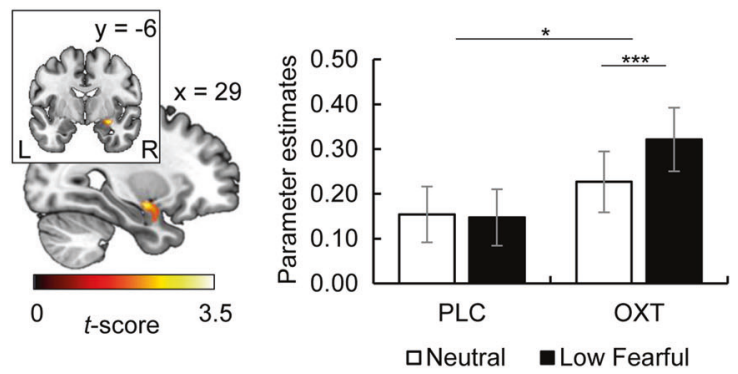

C (Across doses: OXT Low Happy > Neutral $>$ PLC Low Happy $>$ Neutral $)$
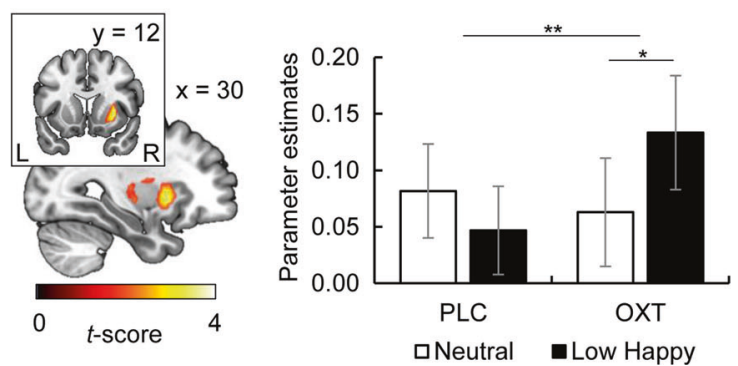

B Amygdala R

$\left(\mathrm{OXT}_{\text {Low Fearful }>\text { Neutral }}>\right.$ PLC Low Fearful > Neutral $)$

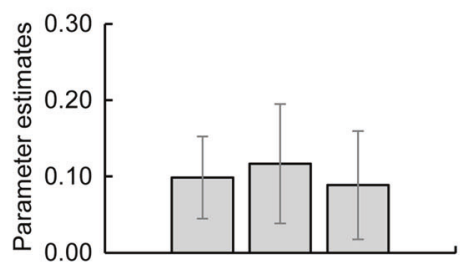

6 IU $12 \mathrm{IU} 24 \mathrm{IU}$

\section{Putamen R}

(OXT Social $>$ PLC Social $)$

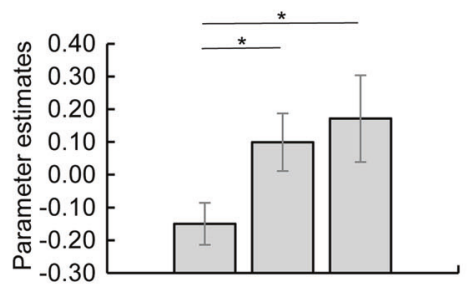

6 IU 12 IU 24 IU

Fig. 2 a Intranasal oxytocin (OXT) significantly enhanced right amygdala responses to low fearful faces compared to neutral faces across doses (interaction of treatment multilpy emotion: $F_{(1,76)}=6.70, p=0.01, \eta_{\mathrm{p}}{ }^{2}=0.08$ ). Post-hoc $t$-tests revealed significantly greater amygdala reactivity to low fearful compared to neutral faces after OXT administration $\left(t_{(78)}=3.75, p<0.001, d=0.15\right)$. b The OXT-induced increase in amygdala activation did not differ between dose groups. $\mathrm{c}$ Intranasal OXT significantly enhanced right putamen responses to low happy faces compared to neutral faces (interaction of treatment multilpy emotion: $F_{(1,76)}=9.13, p=0.003, \eta_{p}{ }^{2}=0.11$ ). Post-hoc $t$-tests revealed significantly greater putamen reactivity to low happy compared to neutral faces after OXT administration $\left(t_{(78)}=2.38, p=0.02, d=0.16\right)$. d The observed OXT effect on right putamen responses to social stimuli across emotional valence (low happy and neutral faces) was more pronounced in the dose groups of 24 and 12 international units (IU) compared to $6 \mathrm{IU}$ (24 IU vs. $6 \mathrm{IU}: t_{(34.87)}=2.19, p=0.04, d=0.60 ; 12 \mathrm{IU}$ vs. $\left.6 \mathrm{IU}: t_{(52)}=2.32, p=0.02, d=0.63\right)$. Error bars indicate standard error of the mean. Abbreviations: IU, international unit; $\mathrm{L}$, left; OXT, oxytocin; PLC, placebo; $\mathrm{R}$, right. ${ }^{*} p<0.05,{ }^{* *} p<0.01$, ${ }^{* * *} p<0.001$.

A (Across doses: OXT Happy > Neutral $>$ PLC Happy > Neutral)

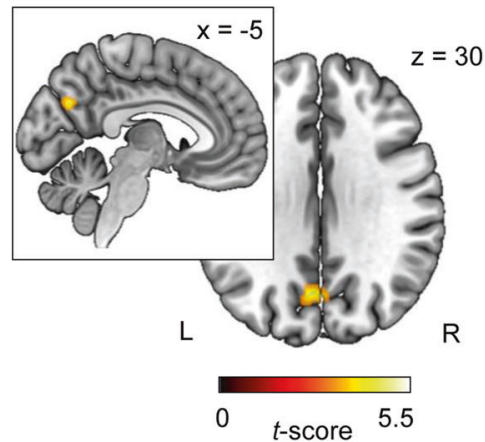

B (Across doses: OXT Happy $>$ Neutral $>$ PLC Happy $>$ Neutral $)$

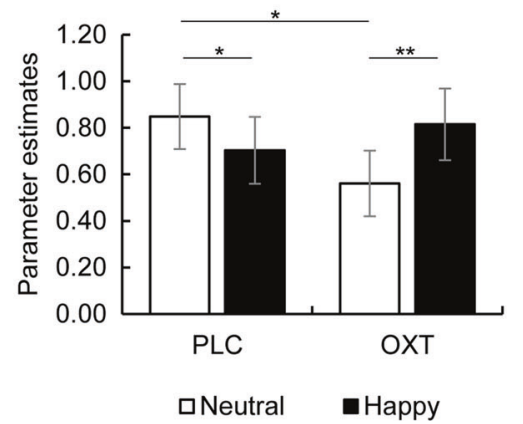

Fig. 3 a Across doses, intranasal oxytocin (OXT) significantly enhanced the responses to happy faces in the left precuneus (Montreal Neurological Institute peak coordinates $\left.x, y, z:-4,-68,30, t_{(78)}=4.34, k=144, p_{\mathrm{FWE}}=0.02\right)$. b Post hoc tests revealed significantly enhanced resposes to happy compared to neutral faces after OXT administration $\left(t_{(78)}=3.40, p=0.001, d=0.19\right)$, whereas precuneus response under placebo (PLC) was greater for neutral faces $\left(t_{(78)}=2.12, p=0.04, d=0.12\right)$. Precuneus reactivity to neutral faces was significantly reduced after OXT administration compared to PLC $\left(t_{(78)}=-2.37, p=0.02, d=-0.23\right)$. Error bars indicate standard error of the mean. Abbreviations: FWE, familywise error corrected; L, left; OXT, oxytocin; PLC, placebo; R, right. ${ }^{*} p<0.05,{ }^{* *} p<0.01$.

fear-specific brain activation or for a dampening OXT effect (e.g. $[$ OXT happy $>$ neutral $]<[$ PLC happy $>$ neutral $]$ ).

Sexual-dimorphic OXT effects. Mixed ANOVAs with sex (female, male) as an additional between-subject factor were calculated for the observed OXT effects on right amygdala activation to low fearful faces, right putamen responses to happy faces across emotional intensities, and left amygdala reactivity to high fearful faces as evident in our previous study [20]. Parameter estimates of groups receiving the same dose of OXT in men and women were used as dependent variables (i.e. 12-IU- and 24-IU-dose groups with a latency of $45 \mathrm{~min}$ ). Results revealed a significant interaction of treatment and sex on right amygdala reactivity $\left(F_{(1,86)}=4.06\right.$, $p=0.047, \eta_{p}{ }^{2}=0.05$; see Fig. $4 a$ ) and interactions of treatment 
A $\left(\mathrm{OXT}_{\text {Social }}>\right.$ PLC $\left._{\text {Social }}\right)$

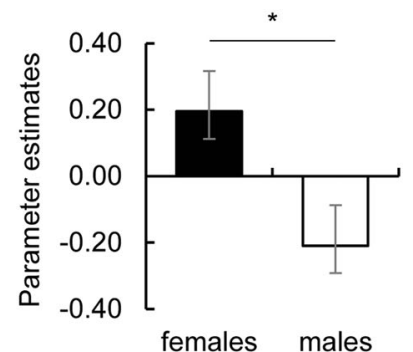

B

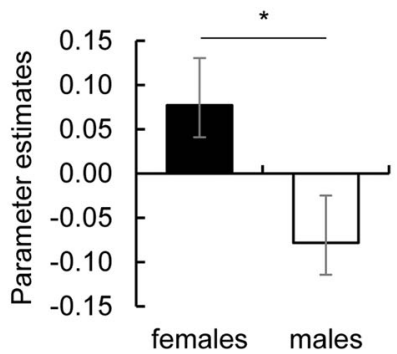

C
Putamen R

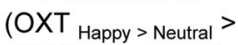

PLC Happy > Neutral $)$

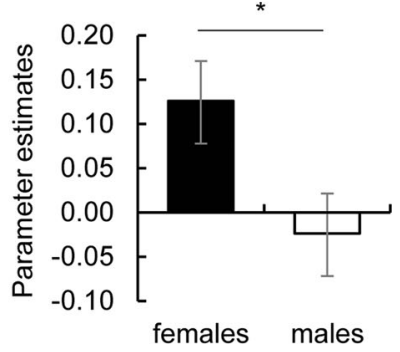

Fig. 4 Effects of intranasal oxytocin (OXT) differed significantly between women and men ([20]; for dose groups of 12 and 24 IU with a latency of $\mathbf{4 5} \mathbf{~ m i n ) . ~ a ~ A c r o s s ~ n e u t r a l ~ a n d ~ l o w ~ f e a r f u l ~ f a c e s , ~ r i g h t ~ a m y g d a l a ~ r e a c t i v i t y ~ w a s ~ e n h a n c e d ~ i n ~ f e m a l e ~ p a r t i c i p a n t s ~ b u t ~ a t t e n u a t e d ~ i n ~}$ male participants (interaction of treatment multilpy sex: $F_{(1,86)}=4.06, p=0.047, \eta_{p}{ }^{2}=0.05$ ). $\mathbf{b}$ Likewise, OXT effects on emotion-specific left amygdala responses to high fearful faces differed between sexes (interaction of treatment multilpy emotion multilpy sex: $F_{(1,86)}=4.74, p=$ $0.03, \eta_{p}{ }^{2}=0.05$ ). c OXT effects on emotion-specific right putamen activation in response to happy faces were significantly more pronounced in women compared to men (interaction of treatment multilpy emotion multilpy sex: $F_{(1,86)}=5.73, p=0.02, \eta_{p}^{2}=0.06$ ). Error bars indicate standard error of the mean. Abbreviations: L, left; OXT, oxytocin; PLC, placebo; R, right. * $p<0.05$.

multilpy emotion multilpy sex on left amygdala response $\left(F_{(1,86)}=\right.$ 4.74, $p=0.03, \eta_{p}{ }^{2}=0.05$; see Fig. $\left.4 \mathrm{~b}\right)$ and right putamen reactivity $\left(F_{(1,86)}=5.73, p=0.02, \eta_{p}{ }^{2}=0.06\right.$; see Fig. $\left.4 c\right)$. Four post hoc twosample $t$-tests were calculated for each significant interaction to compare parameter estimates of baseline contrasts between sexes. Tests showed significantly enhanced right amygdala reactivity to low fearful faces after OXT administration in women compared to men $\left(t_{(68.11)}=2.60, p_{\text {corr }}=0.046, d=0.55\right)$, whereas a trend-to-significant smaller baseline left amygdala activation to high fearful faces $\left(t_{(66.41)}=-2.39, p_{\text {corr }}=0.08\right)$ in women was no longer evident after OXT administration $\left.\left(t_{57.87}\right)=0.75, p_{\text {corr }} \approx 1.00\right)$. In the right putamen, female participants showed attenuated baseline reactivity to neutral $\left(t_{(88)}=-3.07, p_{\text {corr }}=0.01, d=-0.65\right)$ and happy faces $\left(t_{(88)}=-4.46, p_{\text {corr }}<0.001, d=-0.95\right)$. Again, apriori sex differences were reduced after OXT administration, with women still exhibiting smaller responses to neutral faces $\left(t_{(76.66)}=\right.$ $\left.-2.97, p_{\text {corr }}=0.02, d=-0.63\right)$ but not happy faces $\left(t_{(79.84)}=\right.$ $\left.-2.03, p_{\text {corr }}=0.18\right)$. No interactions of treatment and sex with dose were observed (all $F<1.06$, all $p s>0.30$ ), indicating that sexspecific OXT effects do not significantly differ between 12 and 24 IU of OXT. For further comparisons of sexual-dimorphic OXT effects between doses, see Supplementary Results.

\section{DISCUSSION}

The present PLC-controlled, crossover design study sought to investigate dose-dependent OXT effects on reactivity of the amygdala and the brain reward system in healthy women by comparing the modulatory impact of three different doses (6 IU, $12 \mathrm{IU}$, and $24 \mathrm{IU}$ ) of intranasally administered OXT. Results revealed that OXT significantly enhanced amygdala responses to fearful and low fearful faces independent of dosing in the administered range. Furthermore, we found that OXT significantly increased striatal reactivity to happy and low happy faces and that these OXT effects might differ between dose groups, although post-hoc tests for this treatment multilpy dose interaction did not survive correction for multiple comparisons. Given significant differences between the sexes in the observed OXT effects, our data are consistent with the notion that OXT has opposing effects on amygdala reactivity in women and men and that these sex-specific effects are not only a byproduct of the hypothesized dose-response function following lower doses in women. OXT effects on striatal responses to happy faces, on the other hand, were more pronounced in women and striatal sexualdimorphic baseline response patterns vanished after OXT administration.
Our findings suggest that OXT increases the processing of social stimuli in women by strengthening the sensitivity for negative signals in the amygdala and by potentiating reward-related responses in the striatum. Importantly, OXT effects were more pronounced for low intensity emotional faces and absent when the salience of social stimuli was high due to high emotion intensity. Our findings therefore support the social salience hypothesis of OXT [17]. From an evolutionary perspective, OXTinduced increases in social salience may contribute to maternal bonding as stronger intrinsic connectivity within an overlapping network comprising the amygdala and striatal areas has been linked to both mothers' behavioral synchrony with their infants and plasma OXT levels [47]. Importantly, previous studies have reported increased perceived social support [32] and enhanced stress-buffering effects of social support in women after the administration of OXT [48], suggesting that increased OXT signaling may still result in anti-anxiety effects in women when the OXT administration is combined with a supporting intervention. Thus, prosocial effects of OXT in men may primarily operate via anxiolysis, $[1,3-5,8,9,13]$ whereas OXT may promote affiliative efforts and social bonding in women $[6,33]$ in a safe context by enhancing the salience of positive social cues in striatal regions and in the precuneus. Furthermore, in ambiguous situations, enhanced amygdala activation after OXT administration may result in avoidance behavior, but because OXT has been found to signal relationship distress in women [33], enhanced amygdala activation may provide the impetus for social contact to ameliorate stress.

Sexual-dimorphic OXT effects could be driven by interactions between gonadal steroid hormones and OXT. On the one hand, higher baseline levels of progesterone in healthy women are associated with hypersensitivity to negative stimuli in emotion processing brain regions including the amygdala [34, 49-56]. On the other hand, reward sensitivity and reward-related striatal activation seem to be enhanced in the late follicular phase of the menstrual cycle when estradiol levels are high and progesterone levels are low [35, 57-59]. These findings of reduced striatal responses to positive stimuli when progesterone levels are raised might explain the observed attenuated baseline putamen reactivity to happy faces in our female sample compared to male participants, as female participants were tested in the luteal phase when progesterone levels are high [60]. Thus, because women have higher estrogen and progesterone levels than men, a small dose of OXT (i.e. 6 IU) may be sufficient to intensify the sensitivity for potential threats in women. Importantly, however, previous studies, which examined the effects of $24 \mathrm{IU}$ on amygdala 
reactivity to fearful faces in healthy women in the luteal [21] or follicular phase [61], consistently found an increase in amygdala activation, indicating that the direction of the OXT effect does not change with hormonal levels fluctuating across the menstrual cycle.

Another possible explanation for sexual-dimorphic OXT effects might be a difference in the distribution of OXT receptors in women and men, as is evident in rodents [36, 37]. However, no sex differences have been found to date for OXT binding sites in the human brain [62]. Importantly, OXT also binds to receptors of the related nonapeptide arginine vasopressin (AVP), although with lower affinity [63]. Considering the evidence for AVP effects in men, which are missing or even reversed in women $[25,26,64-$ 66], sexually-specific OXT effects might result from an interaction with the AVP system. Therefore, reported dose-dependent effects on amygdala activation in men missing in women might be explained by OXT occupying AVP receptors and producing AVPlike effects at higher OXT doses [20,67].

Furthermore, opposing OXT effects on amygdala reactivity in women and men may be related to different intra-amygdalar targets of OXT. The amygdala is not a single homogenous structure but rather comprises several functionally specialized subnuclei that respond differentially to emotional stimuli [68]. Given a higher sensitivity of the centromedial amygdala to negative emotional stimuli compared to the basal amygdala [68], it is conceivable that OXT might reduce sensitivity of the centromedial amygdala in men while improving the sensitivity of basal nuclei in women. However, future studies with ultra-field imaging are required to reliably differentiate amygdala subnuclei.

Our study has some limitations. First, as we expected a reduction of amygdala activation in women following lower doses, we compared the effects of 24 IU OXT with those of smaller doses. Higher doses than $24 \mathrm{IU}$ might be needed to detect the hypothesized inverted- $U$ relationship between OXT dose and reward-associated brain activation [28] or dose-dependent effects on amygdala activation. Second, we explored the kinetics of OXT in a sample of healthy women, and further patient studies are warranted to extrapolate our findings to psychopathology.

Conclusively, our results provide support for the sexualdimorphic effects of OXT and suggest that OXT increases the salience of social information in women by potentiating limbic and striatal reactivity to low intensity social stimuli. As such, our findings may have important implications for the therapeutic applications of OXT. Strict control of the therapeutic context and the combining of OXT with other anti-anxiety interventions may be required to produce anxiolytic effects in women. Further insights into sex-specific OXT signaling might also broaden our understanding of etiological and pathogenetic mechanisms underlying mental disorders associated with social dysfunctions and substantial sex differences in prevalence rates such as autism spectrum disorder.

\section{FUNDING AND DISCLOSURE}

DS and RH are supported by a German Research Foundation (DFG) grant (SCHE 1913/5-1 and HU 1302/11-1). We report no competing biomedical financial interests or personal affiliations in connection with the content of this paper.

\section{ACKNOWLEDGEMENTS}

We thank Paul Jung for programming assistance and Alexandra Patin for proofreading the paper.

\section{ADDITIONAL INFORMATION}

Supplementary Information accompanies this paper at (https://doi.org/10.1038/ s41386-019-0582-6)
Publisher's note Springer Nature remains neutral with regard to jurisdictional claims in published maps and institutional affiliations.

\section{REFERENCES}

1. Eckstein M, Becker B, Scheele D, Scholz C, Preckel K, Schlaepfer TE, et al. Oxytocin facilitates the extinction of conditioned fear in humans. Biol Psychiatry. 2015;78:194-202.

2. Eckstein M, Scheele D, Patin A, Preckel K, Becker B, Walter A, et al. Oxytocin facilitates pavlovian fear learning in males. Neuropsychopharmacology. 2016;41:932-9.

3. Eckstein M, Scheele D, Weber K, Stoffel-Wagner B, Maier W, Hurlemann R. Oxytocin facilitates the sensation of social stress. Hum Brain Mapp. 2014;35:4741-50.

4. de Oliveira DC, Zuardi AW, Graeff FG, Queiroz RH, Crippa JA. Anxiolytic-like effect of oxytocin in the simulated public speaking test. J Psychopharmacol 2012;26:497-504.

5. Heinrichs M, Baumgartner T, Kirschbaum C, Ehlert U. Social support and oxytocin interact to suppress cortisol and subjective responses to psychosocial stress. Biol Psychiatry. 2003;54:1389-98.

6. Walum $\mathrm{H}$, Young $\mathrm{LJ}$. The neural mechanisms and circuitry of the pair bond. Nat Rev Neurosci. 2018;19:643-54.

7. Feldman R. The neurobiology of human attachments. Trends Cogn Sci. 2017;21:80-99.

8. Kirsch $\mathrm{P}$, Esslinger $\mathrm{C}$, Chen $\mathrm{Q}$, Mier $\mathrm{D}$, Lis $\mathrm{S}$, Siddhanti $\mathrm{S}$, et al. Oxytocin modulates neural circuitry for social cognition and fear in humans. J Neurosci. 2005;25:11489-93.

9. Domes G, Heinrichs M, Gläscher J, Büchel C, Braus DF, Herpertz SC. Oxytocin attenuates amygdala responses to emotional faces regardless of valence. Biol Psychiatry. 2007;62:1187-90.

10. Viviani $D$, Charlet $A$, van den Burg E, Robinet $C$, Hurni N, Abatis $M$, et al. Oxytocin selectively gates fear responses through distinct outputs from the central amygdala. Science. 2011;333:104-7.

11. Knobloch HS, Charlet A, Hoffmann LC, Eliava M, Khrulev S, Cetin AH, et al. Evoked axonal oxytocin release in the central amygdala attenuates fear response. Neuron. 2012;73:553-66.

12. Liu N, Hadj-Bouziane F, Jones KB, Turchi JN, Averbeck BB, Ungerleider LG. Oxytocin modulates $\mathrm{fMRI}$ responses to facial expression in macaques. Proc Natl Acad Sci USA. 2015;112:E3123-30.

13. Kanat M, Heinrichs M, Mader I, van Elst LT, Domes G. Oxytocin modulates amygdala reactivity to masked fearful eyes. Neuropsychopharmacology. 2015;40:2632-8

14. Scheele D, Wille A, Kendrick KM, Stoffel-Wagner B, Becker B, Güntürkün O, et al. Oxytocin enhances brain reward system responses in men viewing the face of their female partner. Proc Natl Acad Sci. 2013;110:20308-13.

15. Scheele D, Plota J, Stoffel-Wagner B, Maier W, Hurlemann R. Hormonal contraceptives suppress oxytocin-induced brain reward responses to the partner's face. Soc Cogn Affect Neurosci. 2016;11:767-74.

16. Kreuder AK, Scheele D, Wassermann L, Wollseifer M, Stoffel-Wagner B, Lee MR, et al. How the brain codes intimacy: the neurobiological substrates of romantic touch. Hum Brain Mapp. 2017;38:4525-34.

17. Shamay-Tsoory SG, Abu-Akel A. The social salience hypothesis of oxytocin. Biol Psychiatry. 2016;79:194-202.

18. Heinrichs M, von Dawans B, Domes G. Oxytocin, vasopressin, and human social behavior. Front Neuroendocrinol 2009;30:548-57.

19. Meyer-Lindenberg A, Domes G, Kirsch P, Heinrichs M. Oxytocin and vasopressin in the human brain: social neuropeptides for translational medicine. Nat Rev Neurosci. 2011;12:524-38.

20. Spengler FB, Schultz J, Scheele D, Essel M, Maier W, Heinrichs M, et al. Kinetics and dose dependency of intranasal oxytocin effects on amygdala reactivity. Biol Psychiatry. 2017;82:885-94.

21. Domes G, Lischke A, Berger C, Grossmann A, Hauenstein K, Heinrichs M, et al. Effects of intranasal oxytocin on emotional face processing in women. Psychoneuroendocrinology 2010;35:83-93.

22. Lischke A, Gamer M, Berger C, Grossmann A, Hauenstein $K$, Heinrichs $M$, et al. Oxytocin increases amygdala reactivity to threatening scenes in females. Psychoneuroendocrinology 2012;37:1431-8.

23. Li K, Nakajima M, Ibañez-Tallon I, Heintz N. A cortical circuit for sexually dimorphic oxytocin-dependent anxiety behaviors. Cell 2016;167:60-72.e11.

24. Dumais KM, Kulkarni PP, Ferris CF, Veenema AH. Sex differences in neural activation following different routes of oxytocin administration in awake adult rats. Psychoneuroendocrinology 2017;81:52-62.

25. Rilling JK, Demarco AC, Hackett PD, Chen X, Gautam P, Stair S, et al. Sex differences in the neural and behavioral response to intranasal oxytocin and 
vasopressin during human social interaction. Psychoneuroendocrinology 2014; 39:237-48

26. Feng C, Hackett PD, DeMarco AC, Chen X, Stair S, Haroon E, et al. Oxytocin and vasopressin effects on the neural response to social cooperation are modulated by sex in humans. Brain Imaging Behav. 2015;9:754-64.

27. Feng C, Lori A, Waldman ID, Binder EB, Haroon E, Rilling JK. A common oxytocin receptor gene (OXTR) polymorphism modulates intranasal oxytocin effects on the neural response to social cooperation in humans. Genes Brain Behav. 2015;14:516-25

28. Borland JM, Rilling JK, Frantz KJ, Albers HE. Sex-dependent regulation of social reward by oxytocin: an inverted $\mathrm{U}$ hypothesis. Neuropsychopharmacology. 2019;44:97-110.

29. Borland JM, Aiani LM, Norvelle A, Grantham KN, O'Laughlin K, Terranova Jl, et al. Sex-dependent regulation of social reward by oxytocin receptors in the ventral tegmental area. Neuropsychopharmacology. 2019;44:785-92.

30. Fischer-Shofty M, Levkovitz $Y$, Shamay-Tsoory SG. Oxytocin facilitates accurate perception of competition in men and kinship in women. Soc Cogn Affect Neurosci. 2013;8:313-7.

31. Scheele D, Striepens N, Kendrick KM, Schwering C, Noelle J, Wille A, et al. Opposing effects of oxytocin on moral judgment in males and females. Hum Brain Mapp. 2014;35:6067-76.

32. Cardoso C, Valkanas $H$, Serravalle L, Ellenbogen MA. Oxytocin and social context moderate social support seeking in women during negative memory recall. Psychoneuroendocrinology 2016;70:63-69.

33. Taylor SE. Tend and befriend: biobehavioral bases of affiliation under stress. Curr Dir Psychol Sci. 2006;15:273-7.

34. van Wingen GA, van Broekhoven F, Verkes RJ, Petersson KM, Bäckström T, Buitelaar JK, et al. Progesterone selectively increases amygdala reactivity in women. Mol Psychiatry. 2008;13:325-33.

35. Dreher JC, Schmidt PJ, Kohn P, Furman D, Rubinow D, Berman KF. Menstrual cycle phase modulates reward-related neural function in women. Proc Natl Acad Sci USA. 2007;104:2465-70.

36. Smith CJ, Poehlmann ML, Li S, Ratnaseelan AM, Bredewold R, Veenema AH. Age and sex differences in oxytocin and vasopressin $\mathrm{V} 1 \mathrm{a}$ receptor binding densities in the rat brain: focus on the social decision-making network. Brain Struct Funct. 2017;222:981-1006.

37. Dumais KM, Bredewold $\mathrm{R}$, Mayer TE, Veenema AH. Sex differences in oxytocin receptor binding in forebrain regions: correlations with social interest in brain region- and sex- specific ways. Horm Behav 2013;64:693-701.

38. Insel TR. Translating oxytocin neuroscience to the clinic: a National Institute of Mental Health perspective. Biol Psychiatry. 2016;79:153-4.

39. Engel S, Laufer S, Miller R, Niemeyer H, Knaevelsrud C, Schumacher S. Demographic, sampling- and assay-related confounders of endogenous oxytocin concentrations: a systematic review and meta-analysis. Front Neuroendocrinol 2019;54:100775.

40. Sheehan DV, Lecrubier $Y$, Sheehan $\mathrm{KH}$, Amorim $\mathrm{P}$, Janavs J, Weiller $\mathrm{E}$, et al. The Mini-International Neuropsychiatric Interview (MINI): the development and validation of a structured diagnostic psychiatric interview for DSM-IV and ICD-10. J Clin Psychiatry. 1998;59:22-33.

41. Watson D, Clark LA, Tellegen A. Development and validation of brief measures of positive and negative affect: the PANAS scales. J Pers Soc Psychol. 1988;54:1063-70.

42. Spielberger C, Gorsuch R, Lushene R, Vagg P, Jacobs G. Manual for the State-Trait Anxiety Inventory. Palo Alto: Consulting Psychologists Press; 1983.

43. Guastella AJ, Hickie IB, McGuinness MM Otis M, Woods EA, Disinger $H M$, et al. Recommendations for the standardisation of oxytocin nasal administration and guidelines for its reporting in human research. Psychoneuroendocrinology 2013;38:612-25

44. Stocker T, Kellermann T, Schneider $F$, Habel $U$, Amunts $K$, Pieperhoff $P$, et al. Dependence of amygdala activation on echo time: results from olfactory fMRI experiments. Neuroimage 2006;30:151-9.

45. Maldjian JA, Laurienti PJ, Kraft RA, Burdette JH. An automated method for neuroanatomic and cytoarchitectonic atlas-based interrogation of $\mathrm{FMRI}$ data sets. Neuroimage 2003;19:1233-9.

46. Maldjian JA, Laurienti PJ, Burdette JH. Precentral gyrus discrepancy in electronic versions of the Talairach atlas. Neuroimage 2004;21:450-5.
47. Atzil S, Touroutoglou A, Rudy T, Salcedo S, Feldman R, Hooker JM, et al. Dopamine in the medial amygdala network mediates human bonding. Proc Natl Acad Sci USA. 2017;114:2361-6.

48. Scheele D, Lieberz J, Goertzen-Patin A, Engels C, Schneider L, Stoffel-Wagner B, et al. Trauma disclosure moderates the effects of oxytocin on intrusions and neural responses to fear. Psychother Psychosom 2019;88:61-63.

49. Conway CA, Jones BC, DeBruine LM, Welling LL, Law Smith MJ, Perrett DI, et al. Salience of emotional displays of danger and contagion in faces is enhanced when progesterone levels are raised. Horm Behav 2007;51:202-6.

50. Protopopescu X, Pan H, Altemus M, Tuescher O, Polanecsky M, McEwen B, et al. Orbitofrontal cortex activity related to emotional processing changes across the menstrual cycle. Proc Natl Acad Sci USA. 2005;102:16060-5.

51. Andreano JM, Cahill L. Menstrual cycle modulation of medial temporal activity evoked by negative emotion. Neuroimage 2010;53:1286-93.

52. Ossewaarde L, Hermans EJ, van Wingen GA, Kooijman SC, Johansson IM, Bäckström T, et al. Neural mechanisms underlying changes in stress-sensitivity across the menstrual cycle. Psychoneuroendocrinology 2010;35:47-55.

53. Derntl B, Hack RL, Kryspin-Exner I, Habel U. Association of menstrual cycle phase with the core components of empathy. Horm Behav 2013;63:97-104.

54. Gingnell M, Morell A, Bannbers E, Wikström J, Sundström Poromaa I. Menstrual cycle effects on amygdala reactivity to emotional stimulation in premenstrual dysphoric disorder. Horm Behav 2012;62:400-6.

55. Derntl B, Kryspin-Exner I, Fernbach E, Moser E, Habel U. Emotion recognition accuracy in healthy young females is associated with cycle phase. Horm Behav 2008;53:90-95.

56. Kamboj SK, Krol KM, Curran HV. A specific association between facial disgust recognition and estradiol levels in naturally cycling women. PLoS One. 2015;10: e0122311.

57. Frank TC, Kim GL, Krzemien A, Van Vugt DA. Effect of menstrual cycle phase on corticolimbic brain activation by visual food cues. Brain Res. 2010;1363:81-92.

58. Diekhof EK, Ratnayake M. Menstrual cycle phase modulates reward sensitivity and performance monitoring in young women: preliminary fMRI evidence. Neuropsychologia 2016;84:70-80.

59. Franklin TR, Jagannathan K, Wetherill RR, Johnson B, Kelly S, Langguth J, et al. Influence of menstrual cycle phase on neural and craving responses to appetitive smoking cues in naturally cycling females. Nicotine Tob Res. 2015;17:390-7.

60. Reed BG, Carr BR. The normal menstrual cycle and the control of ovulation. In: Feingold KR, Anawalt B, Boyce A, Chrousos G, Dungan K, Grossman A, et al., editors. Endotext. South Dartmouth (MA): MDText.com, Inc.; 2000.

61. Lischke A, Herpertz SC, Berger C, Domes G, Gamer M. Divergent effects of oxytocin on (para-)limbic reactivity to emotional and neutral scenes in females with and without borderline personality disorder. Soc Cogn Affect Neurosci. 2017;12:1783-92.

62. Quintana DS, Rokicki J, van der Meer D, Alnæs D, Kaufmann T, Córdova-Palomera A, et al. Oxytocin pathway gene networks in the human brain. Nat Commun. 2019;10:668

63. Cirillo R, Gillio Tos E, Schwarz MK, Quattropani A, Scheer A, Missotten M, et al. Pharmacology of (2S,4Z)-N-[(2S)-2-hydroxy-2-phenylethyl]-4-(methoxyimino) -1[(2'-methyl[1,1'-biphenyl]-4-yl)carbonyl]-2-pyrrolidinecarboxamide, a new potent and selective nonpeptide antagonist of the oxytocin receptor. J Pharmacol Exp Ther. 2003;306:253-61.

64. Chen X, Hackett PD, DeMarco AC, Feng C, Stair S, Haroon $E$, et al. Effects of oxytocin and vasopressin on the neural response to unreciprocated cooperation within brain regions involved in stress and anxiety in men and women. Brain Imaging Behav. 2016;10:581-93.

65. Motoki K, Sugiura M, Takeuchi H, Kotozaki Y, Nakagawa S, Yokoyama R, et al. Are plasma oxytocin and vasopressin levels reflective of amygdala activation during the processing of negative emotions? A preliminary study. Front Psychol 2016;7:480.

66. Thompson RR, George K, Walton JC, Orr SP, Benson J. Sex-specific influences of vasopressin on human social communication. Proc Natl Acad Sci USA. 2006;103: 7889-94.

67. Brunnlieb C, Münte TF, Tempelmann C, Heldmann M. Vasopressin modulates neural responses related to emotional stimuli in the right amygdala. Brain Res. 2013;1499:29-42.

68. Hrybouski S, Aghamohammadi-Sereshki A, Madan CR, Shafer AT, Baron CA, Seres $P$, et al. Amygdala subnuclei response and connectivity during emotional processing. Neuroimage 2016;133:98-110. 